\title{
Improved Executive Functions and Reduced Craving in Youths with Methamphetamine Addiction: Evidence from Combined Transcranial Direct Current Stimulation with Mindfulness Treatment
}

\author{
Jaber Alizadehgoradel ${ }^{1}$, Saeed Imani ${ }^{2}$, Vahid Nejati ${ }^{3}$, Marie-Anne Vanderhasselt ${ }^{4,5}$, Behnam Molaei ${ }^{6}$, \\ Mohammad Ali Salehinejad ${ }^{7,8}$, Shirin Ahmadi ${ }^{9}$, Mina Taherifard ${ }^{9}$ \\ ${ }^{1}$ Department of Clinical and Health Psychology, ${ }^{2}$ Department of Clinical and Health Psychology \& Counseling Group, Shahid Beheshti \\ University, ${ }^{3}$ Department of Clinical and Health Psychology, Faculty of Education \& Psychology, Shahid Beheshti University, Tehran, Iran, \\ ${ }^{4}$ Department of Experimental Clinical and Health Psychology, Ghent University, ${ }^{5}$ Department of Head and Skin, Psychiatry and Medical \\ Psychology, Ghent University Hospital, Ghent, Belgium, ${ }^{6}$ Department of Psychiatry, Faculty of Medicine, Ardabil University of Medical Sciences, \\ Ardabil, Iran, ${ }^{7}$ Department of Psychology and Neurosciences, Leibniz Research Centre for Working Environment and Human Factors, Dortmund, \\ ${ }^{8}$ Ruhr-University Bochum, International Graduate School of Neuroscience, Bochum, Germany, ${ }^{9}$ Department of Psychology, Mohaghegh-Ardabili \\ University, Ardabil, Iran
}

Objective: Transcranial direct current stimulation (tDCS) and mindfulness practices have been proposed as a potential approach to improve executive functions (EFs) and reduce craving in persons with substance use disorders. Based on the neural mechanisms of action of each of these interventions, the combination of both non-pharmacological interventions might have additive effects. In the current study, the effects of tDCS combined with mindfulness-based substance abuse treatment (MBSAT) to improve EFs and reduce craving were investigated in early abstinent methamphetamine abuse.

Methods: Eighty (youths aged between 18 and 21) early-abstinent methamphetamine users were randomly assigned to the research groups (tDCS group $[\mathrm{n}=20]$, mindfulness group $[\mathrm{n}=20]$, combined mindfulness-tDCS group [n $=$ $20]$, and sham group [ $\mathrm{n}=20])$. Active tDCS $(1.5 \mathrm{~mA}, 20 \mathrm{~min}, 12$ sessions) or sham tDCS was appliedover the left dorsolateral prefrontal cortex and the MBSAT protocol was used over twelve 50-min sessions.

Results: Both in the post-test phase (immediately after the intervention) and follow-up phase (one month after the intervention), performance in most EFs tasks significantly improved in the combination group which received real tDCS + MBSAT, as compared to baseline values and sham stimulation group. Similarly, a significant reduction in craving was observed after intervention inall treatment groups, but not the sham stimulation group. Interestingly, the increase in EFs and the reduction in craving post versus pre tDCS + MBSAT intervention were correlated.

Conclusion: Findings from the current study provide initial support for the clinical effectiveness of combination tDCS + MBSAT, possibly influencing cognitive/affective processes.

KEY WORDS: Executive functions; Transcranial direct current stimulation; Mindfulness; Substance-related disorders; Youths.

Received: August 22, 2020 / Revised: October 26, 2020

Accepted: November 1, 2020

Address for correspondence: Jaber Alizadehgoradel

Department of Clinical and Health Psychology, Faculty of

Psychology and Educational Sciences, Shahid Beheshti

University, P.O. Box: 1983963113, 193954716, Tehran, Iran

E-mail: j.alizadeh45@gmail.com

ORCID: https://orcid.org/0000-0003-4803-0607

Saeed Imani

Department of Clinical and Health Psychology \& Counseling

Group, Shahid Beheshti University, P.O. Box: 1983963114,

193954717, Tehran, Iran

E-mail: s_imani@sbu.ac.ir

ORCID: https://orcid.org/0000-0001-5290-2995

\section{INTRODUCTION}

Drug abuse is one of the most widespread and costly health problems in modern societies and customary medical treatments are often ineffective and relapse is prevalent [1]. Biological findings of Johnston et al. [2] have indicated that the prevalence of substance abuse increases with age during adolescence and increases in early adulthood. The unprecedented progress of neuroscientifi cprocedures has resulted in imperative neuropsychological

(c) This is an Open-Access article distributed under the terms of the Creative Commons Attribution Non-Commercial License (http://creativecommons.org/licenses/by-nc/4.0) which permits unrestricted non-commercial use, distribution, and reproduction in any medium, provided the original work is properly cited. 
models, for which the combination of neurobiological outcomes and psychological processes provide a better understanding of the onset, maintenance and relapse of substance use disorders [3].

Studies conducted in the past decade and meta-analyses have determined arelationship between substance use and cognitive deficits [4]. It has been established that, in adolescents, problems in executive functions (EFs) increase the risk of substance use and other potentially addictive behaviors such as overeating andcomputer game addiction $[5,6]$. Misuse of nicotine during adolescence can increase EFs problems by mediating with the growthof neural connectivity to prevent impulsive limbic signals $[7,8]$. Reciprocally, training adolescents in EFs skills early onproduce long-term effects on preventing cigarette and alcohol use and reduce impulsive behavior as an intermediate factor of risky health behaviours [9]. In recent years, some studies in this area have examined the relationship between the executive functions and the consequences of treatment [10].

Neurobiological studies of addiction have established a relationshipbetween the rewarding and boosting effects of drugs and the activation of several areas in the mesocorticolimbic network including the nucleus accumbens and the ventral tegmentalarea with the dopaminergic transmission in the orbitofrontal cortex, dorsolateral prefrontal cortex (DLPFC), and anterior cingulate cortex [11]. New neurological studies such as Hartwell et al. [12] which adopt a brain imaging approach,show that the DLPFC is a core component of the neural substrate in different drug cravings. The DLPFC is most closely related to EFs [13].

One of the main techniques of non-invasive brain stimulation to modulate brain activity and cortical excitability is transcranial direct current stimulation (tDCS) which involves the use of a low-intensity electrical current over the scalp $[14,15]$. Recently, researchers have applied tDCS in children and adolescents with various neuropsychiatric disorders [16-18]. This nascent literature addressesboth safety and potential therapeutic efficiency in children and adolescents $[19,20]$. Recently, the interest in non-invasive methods of stimulating brain activity in the treatment of addiction has increased [21-23]. tDCS has been used in numerous studies for exploring cognitive functions $[24,25]$, including executive functions in healthy persons $[26,27]$ and clinical populations with executive dysfunc- tions $[28,29]$.

Studies using tDCS over the left DLPFC have demonstrated significant improvement in executive functions in people with substance use disorder [30-33]. In addition, anodal tDCS applied over the DLPFC has been widely used in the treatment of substance use disorder (SUD) including methamphetamine dependency $[34,35]$. As described above, this area is involved in spontaneous and cue-elicited craving and EFs related to addictive behaviors (i.e., decision making, response inhibition, risk-taking, attentional bias) [36]. Although tDCS can be effectively used as an alternative therapy, new studies have proposed it as an "augmentative treatment" to promote the effects of other psychotherapy treatments $[37,38]$. Studies have also reported a significant increase in the effectiveness of tDCS when combined with other psychological interventions [39]. Nejati et al. [40] proposed a novel treatment strategy called Psychologicallntervention Combined with Direct Electrical Stimulation to investigate the efficacy of tDCS combined with psychotherapy for major depression treatment. This approach can be used for other psychological interventions combined with neuromodulatory interventions.

Today, mindfulness training is considered a promising treatment for substance abuse [41]. The mindfulness efficacy studies in adolescence and youths are growing rapidly. Mindfulness involves directing attention to the experience of the present moment, anawareness accompanied by an attitude of nonjudgment or acceptance fostered in its practice [42]. A review of studies shows that mindfulness practice has a direct influence on cognitive processes, such as executive functioning [43]. Empirical evidence suggests that mindfulness-based interventions (MBIs) improve substance use disorders by increasing the cognitive regulation of several activity processes, including making clear cognitive appraisals and moderating negative emotions to decrease permanent cognition and emotional arousal; increasing metacognitive awareness to reducedrug-related attentional bias; reducing cue reactivity and increasing cognitive control over craving [44]. All in all, both mindfulness and tDCS have independently benefits for SUD treatment. MBIs have been piloted with adolescents and youthsdealing with substance use disorders [45]. Results of studies in this field have confirmed the applicability of mindfulness interventions to adolescents and youths with substance abuse 
disorders $[45,46]$.

Further, there is evidence from recent studies thattDCS of the frontal cortex can enhance the effects of mindfulness practice by improving working memory [47], and increasing positive affective [48], or increasing awareness [49]. Also, the results of a study by Monnart et al. [50] showed that the combination of tDCS method with mindfulness has led to improved symptoms of depression in people resistant to treatment. The combination of mindfulness with tDCS may provide synergistic benefits to reducing symptoms of SUD greater than each can provide alone [51] but has not studied yet.

In the present study, we evaluated the effects of mindfulness (as a psychological intervention), tDCS, and the combination of tDCS and mindfulness-based substance abuse treatment (tDCS/MBSAT) to improve executive functions and reduce craving in youths early abstinent with methamphetamine use disorders. Also, we used a psychological intervention package consisting of MBSAT, as an effective therapy for adolescents with substance use disorders [52]. It is assumed that tDCS + MBSATis more effective in the improvement of different kinds of executive functions and craving in youths with methamphet- amine dependence. Also, we expect tDCS + MBSAT to have longer-term effects compared to other interventions, especially tDCS. To our knowledge, thisis the first study investigating the effectiveness of the combination of psychological interventionand tDCS using a full-factorial design on the improvement of a battery of executive functions (e.g., response inhibition, risk-taking, cognitive flexibility, and working memory) and craving in youths with methamphetamine dependence. Also, in the present study, we want to find out whether the improvement of executive functions leads to a decrease in cravings or not?

\section{METHODS}

\section{Participants}

Eighty youths boys aged 18 to 21 with early abstinent methamphetamine abuse were enrolled in the study (mean age $=19.46$, standard deviation $=1.15)$. The inclusion criteria were: (1) referral to the addiction treatment center in Ardabilfor quitting methamphetamine use, (2) Methamphetamine use disorder diagnosis based on a clinical interview by an experienced psychiatrist according to the DSM-IV criteria, (3) a history of at least 12

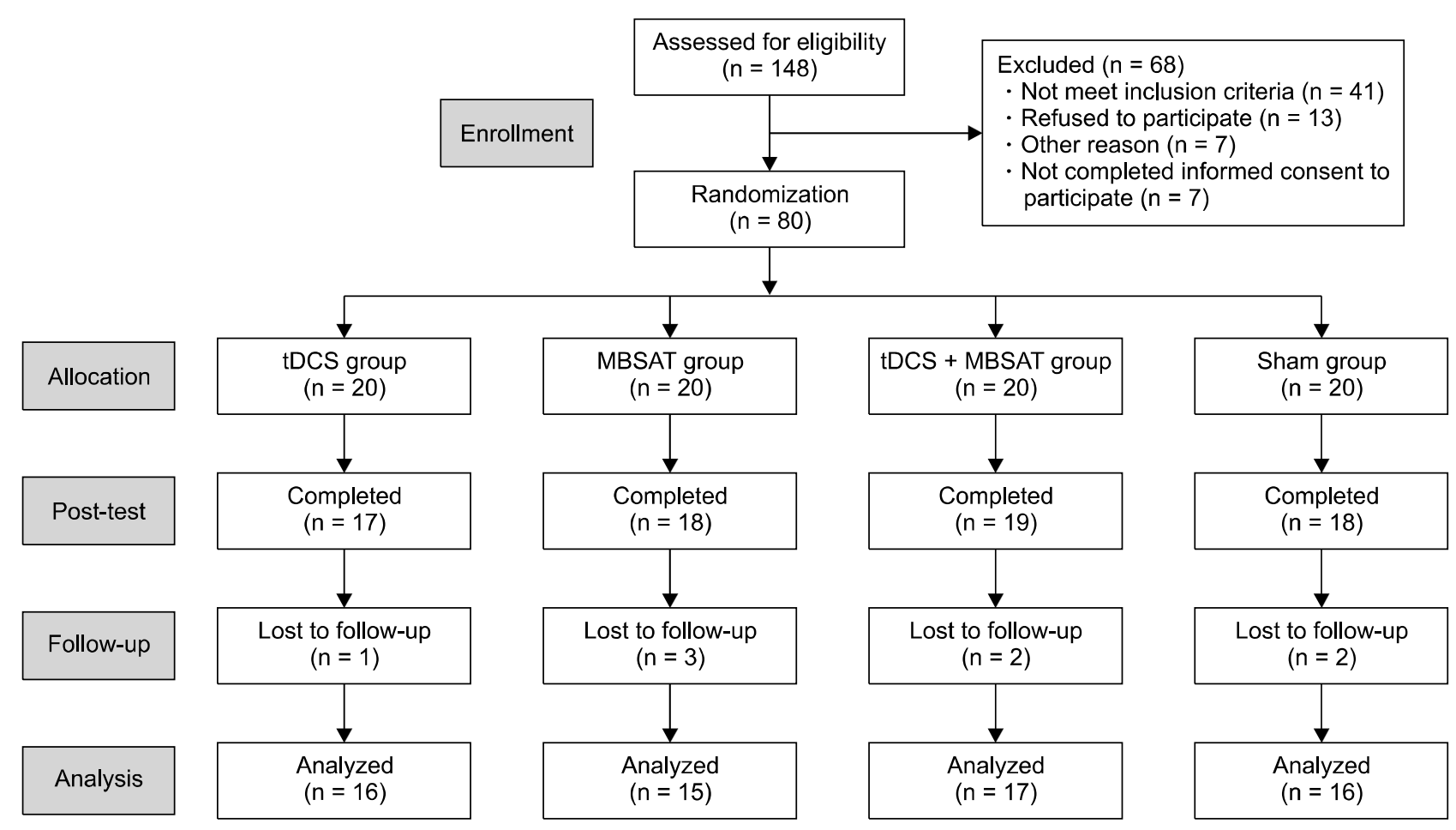

Fig. 1. Flowchart of study inclusion.

tDCS, transcranial direct current stimulation; MBSAT, mindfulness-based substance abuse treatment. 
months methamphetamine use before referral to the addiction treatment center, (4) age range of 18 to 21 years, (5) abstinence from any sedative or stimulant drug except for nicotine, as confirmed by a negative urine test, (6) not being on antidepressants or other psychotropic medications during the study. Exclusion criteria were: (1) inability to tolerate tDCS, (2) comorbidity with axis I disorders such as bipolar disorder, schizophrenia, and any axis II disorders, (3) cranial or brain metal implant, (4) history of seizures, epilepsy or brain injury and any neurological disorders. Four subjects from the tDCS group, four subjects from the sham group, three subjects from the combination group, and five subjects from the MBSAT group could not complete the whole treatment, and thus our final analysis was conducted on 64 participants (Fig. 1). Demographic information of the youths with methamphetamine abuse is shown in Table 1.

\section{Procedures}

Eighty youthboys with methamphetamine use disorderwere initially recruited from the Azadi Rehabilitation Centre for Addiction in Ardabil, Iran and were randomly assigned to the tDCS, MBSAT, tDCS + MBSAT and sham stimulation groups by the block randomization method. We used the simple randomization methods centrally and randomization sequence was generated using an online randomization software (www.sealedenvelope.com). All participants completed the measures three times: before treatment (pre-test), after treatment (post-test), and one month after treatment (follow-up). In the tDCS group, participants received 12 sessions of 20-minute stimulation (two sessions weekly) in 6 weeks with a 72-hour interval between sessions. The MBSAT group participated in the protocol mindfulness consisting of 12 sessions (two sessions weekly). Each MBSAT session lasted 45-50 minutes. Finally, participants in the tDCS + MBSAT group received 12 sessions of 20-minute tDCS (two sessions weekly) and right after each tDCS sessionfollowed the MBSAT session. The sham group received 12 sessions of 20-minute (two sessions weekly) in 6 weeks with a 72-hour interval between sessions. After the end of the study, youths participating in this study were able to participate in complementary psychological treatment. Participants in the sham stimulation group received real stimulation after the end of the study. The current study complied with the ethical principles set forth in the latest edition of the Declaration of Helsinki, and it is approved by the ethical committee of Shahid Beheshti University (no. IR.SBU. ICBS.97/1036). Subjects granted consent before participation and were free to withdraw at any point during the study. All participants in this study were able to participate in the treatment after the end of the study. Also, this study is registered at the Iranian Registry of Clinical Trials, registration number IRCTID: IRCT20181013041327N1.

\section{Intervention}

\section{Mindfulness-based substance abuse treatment}

The psychological intervention used in the present study consisted of mindfulness-based substance abuse treatment [52]. The MBSAT protocol used in this study consisted of twelve 50-minute sessions, two sessions per

Table 1. Demographic data

\begin{tabular}{lccccc}
\hline \multicolumn{1}{c}{ Variable } & tDCS & MBSAT & tDCS + MBSAT & Sham & $p$ value* \\
\hline Sample size & 16 & 15 & 17 & 16 & 0.995 \\
Age & $19.43 \pm 1.20$ & $19.46 \pm 1.12$ & $19.52 \pm 1.23$ & $19.43 \pm 1.15$ \\
Sex, male/female & $16 / 0$ & $15 / 0$ & $17 / 0$ & $16 / 0$ & 0.861 \\
Marital status, single/married & $11 / 5$ & $11 / 4$ & $12 / 5$ & $13 / 3$ & 0.812 \\
Length of methamphetamine use & $3.06 \pm 0.77$ & $3.13 \pm 1.06$ & $2.88 \pm 0.99$ & $2.87 \pm 0.89$ \\
Age of onset of substance use & $15.62 \pm 1.40$ & $15.26 \pm 2.15$ & $15.88 \pm 1.61$ & $15.00 \pm 2.12$ & 0.541 \\
Substance use by family members, yes/no & $8 / 8$ & $6 / 9$ & $11 / 6$ & $9 / 7$ & 0.556 \\
Education & & & & 11 & 0.633 \\
$\quad$ Under the diploma & 10 & 9 & 9 & 5 & \\
$\quad$ Diploma & 6 & 6 & & & \\
\hline
\end{tabular}

Values are presented as number $(\%)$ or mean \pm standard deviation.

tDCS, transcranial direct current stimulation; MBSAT, mindfulness-based substance abuse treatment.

*To analyze the differences between groups in the demographic variables, chi-square analysiscategorical variables andtestscontinuous variables were used. 
week. The structure and contents of each session are shown in Table 2.

\section{tDCS protocol}

The tDCS device in use was the battery-powered “ActivaDose II lontophoresis" Delivery Unit manufactured by ActivaTek, with a 9-V battery as its power source. In the active tDCS adolescent sreceived stimulation for 20 minutes (and 30 seconds in the sham tDCS) at $1.5 \mathrm{~mA}$ intensity with 72-hour intervals between sessions.in tDCS, Combination (tDCS with mindfulness) and sham group the anode electrode was fixed over area F3 (IDLPFC), whereas the cathode electrode was positioned over area F4 (rDLPFC) according to the 10-20 EEG electrode systems. In the sham condition, the electrical current was ramped up for 30 seconds and the current intensity was decreased to $0 \mathrm{~mA}$ without the participants' knowledge. After each tDCS session (active or sham) side effects were evaluated.

\section{Materials}

\section{$\mathrm{N}$-Back test}

This test measures the working memory [53], and is one of the most frequently used culture-independent tests. Several visual stimuli appear on the screen sequentially. Participants asked to press " 1 " if the stimulus on the screen is identical to the previous one, and to press " 2 " if it is different. In this task, the participants were required to store the information for only one stimulus (i.e., the stim-

Table 2. Structure and guideline of each MBSAT session [52]

\begin{tabular}{|c|c|}
\hline $\begin{array}{l}\text { MBSAT } \\
\text { session }\end{array}$ & The theme of mindfulness-based substance abuse treatment (MBSAT) session \\
\hline Session 1 & $\begin{array}{l}\text { Introduction to the Program: First session includes the following: 1) Informal Greeting. 2) Introduction to the Program. } \\
\text { 3) Agreements of group. 4) Defining Mindfulness. 5) Meditation: Mindfulness of Deep Breathing. 6) Group Poll: Learning } \\
\text { Interests of the Youth. 7) Homework and Close-Out. }\end{array}$ \\
\hline Session 2 & $\begin{array}{l}\text { Mindfulness of Drugs and Their Health Effects: Session } 2 \text { includes the following: 1) Centering Meditation. 2) Mindful Check-In. } \\
\text { 3) Drug Classifications Activity. 4) Fatal Drug Combinations. 5) Meditation: Mindfulness of Deep Breathing. 6) Homework and } \\
\text { Close-Out. }\end{array}$ \\
\hline
\end{tabular}

Session 3 Reacting vs. Responding: Session 3 includes the following: 1) Role Play: Mental vs. Physical Power. 2) Discussion: Reaction vs. Response. 3) STIC (Stop, Take a breath, Imagine the future consequences, Choose) Contemplation. 4) STIC Role-Plays. 5) Meditation: Mindfulness of the Breath. 6) Mindful Check-In. 7) Homework and Close-Out.

Session 4 Mindfulness of Delusion: Session 4 includes the following: 1) Centering Meditation. 2) Poem: “The Perfect High. 3) Mindful Check-In. 4) Debate: Pros and Cons of Substance Use. 5) Personal Pros and Cons of Substance Use. 6) Meditation: Bodyscan. 7) Homework and Close-Out.

Session 5 Emotional Awareness: Ssession 5 includes the following: 1) Centering Meditation: Bodyscan. 2) Emotional Categories. 3) Emotional Expression and Gender Norms. 4) Stand If. 5) Deep Disclosure. 6) Game: Concentration. 7) Homework and Close-Out.

Session 6 The Brain and Drugs: Session 6 includes the following: 1) Youth-Led Centering Meditation. 2) Mindful Check-In. 3) Brain Presentation I. 4) Meditation Break. 5) Brain Presentation II: Substance Use, Trauma, and the Mindful Brain. 6) Meditation: Bodyscan. 7) Homework and Close-Out.

Session $7 \quad$ Mindfulness of Craving: Session 7 includes the following: 1) Youth-Led Centering Meditation. 2) Mindful Check-In. 3) Mindful Eating Activity. 4) The Role of Craving in Drug Use. 5) Nonmoving Bodyscan. 6) Worksheet: Roots of Craving. 7) Homework and Close-Out.

Session 8 Mindfulness of Triggers: Session 8 includes the following: 1) Youth-Led Centering Meditation. 2) Mindful Check-In. 3) Mindfulness of Triggers. 4) Three Levels of Influence. 5) Meditation: Noting Awareness. 6) Homework and Close-Out.

Session 9 The Family System and Drugs: Session 9 includes the following: 1) Youth-Led Centering Meditation. 2) My Children Contemplation. 3) The Effect of Drug Use on Family Relationships. 4) Addiction and Intergenerational Trauma. 5) Meditation: Compassion for Family Members. 6) Mindful Check-In. 7) Homework and Close-Out.

Session 10 Mindfulness of the Peer System: Session 10 includes the following: 1) Peer Pressure Role-Play. 2) Discussion: Friends vs. Accomplices. 3) Mindful Check-In With Prompt. 4) Mindful Communication. 5) Youth Developed Peer Pressure Role-Plays. 6) Meditation: Compassion for Friends and Accomplices. 7) Homework and Close-Out.

Session 11 Mindfulness of the External Environment: Session 11 includes the following: 1) Youth-Led Centering. 2) Mindful Check-In. 3) Mindfulness of External Environment. 4) Transforming Systems of Influence. 5) Meditation: Compassion Meditation Toward Community. 6) Homework and Close-Out.

Session 12 Closing Ceremony: Session 12 includes the following: 1) Meditation: Final Practice. 2) Mindful Check-In. 3) Focus Group. 4) Group Appreciations. 5) Pizza Party/Food Celebration. 6) Certificates of Completion. 7) Closing Dedication and Ceremony. 
ulus right before the present stimulus). In addition, the replacement of the previous stimulus by a new one means that the participant needs to update the information stored in memory. This task is designed in such a way that it makes responding to all stimuli at all levels mandatory. Thus, completing it requires constant control and updating of the information in the working memory. The task contains a set of one hundred linear images. We used the number of correct responses and reaction time to assess working memory. This task is widely used in clinical and empirical studies, and its high validity has been proved through association with several other working memory tasks [54] (Fig. 2).

\section{Wisconsin Card Sorting Test (WCST)}

WCST is one of the main tools used for assessing the performance of the prefrontal cortex, specifically the DLPFC [55]. Demakis [55] have also emphasized this task's usability as a frontal cortex measure. The present study used a computerized version of the task. WCST in-
A

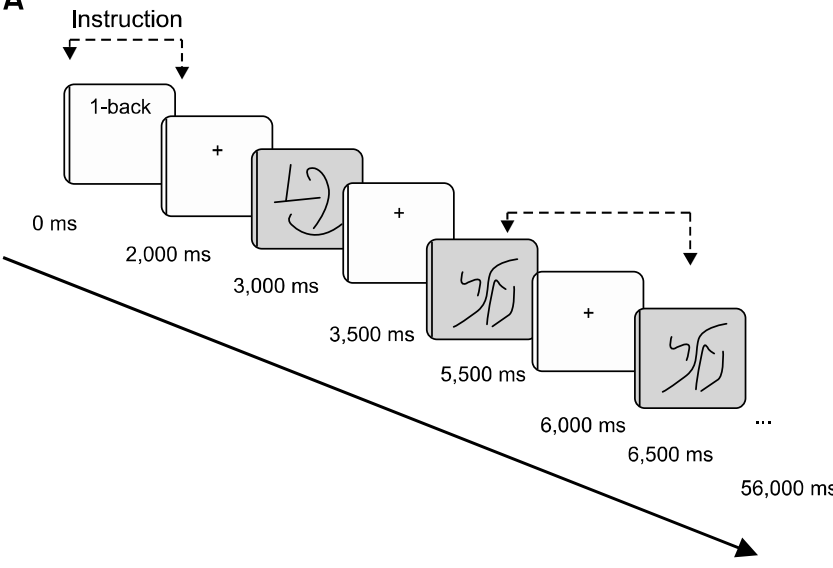

B
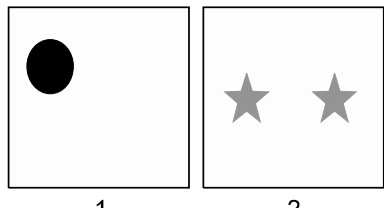

2
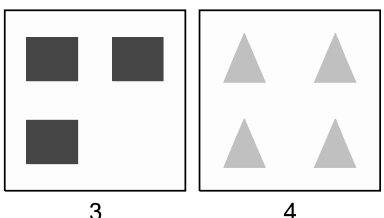

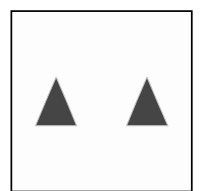

\section{2}

Press key

C

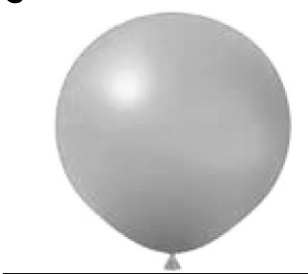

Pump up the balloon

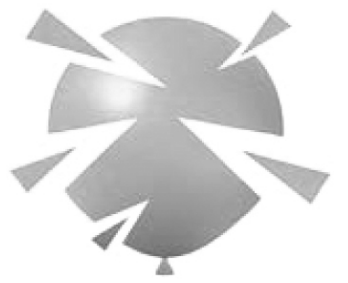

Pump up the balloon
Earning from previous trial $=\$ 2$ Potential earnings $=\$ 5$ Number of pumps $=43$

Total earnings (account) $=\$ 7$ Balloon number $=2$ of 30

Collect the money
Earning from previous trial $=\$ 10$ Potential earnings $=\$ 0$ Number of pumps $=80$

Total earnings (account) $=\$ 10$ Balloon number $=7$ of 30

Fig. 2. Pictures of the executive function tasks: The 1-back (A), WCST (B), BART (C) and Go-No-Go (D). All computerized tasks were presented on a 15.6 " screen in counterbalanced order.

WCST, Wisconsin Card Sorting Task; BART, Balloon Analogue Risk Task. 
cludes 64 dissimilar cards with four types of shapes (triangular, star, cross, and circle) printed on them. Each card can be one of four colors (blue, red, yellow, or green). In short, the test has three sorting rules: shape, number (amount), and color. By receiving a "correct" or "wrong" feedback, they discover the sorting rule. After putting the card in the correct category, the rule changes and participants have to discover the new rule based on the feedback they receive. The participants are scored by the number of ten-card categories they sorted successfully. If a participant continues to sort by the last sorting rule while the rule has changed, a preservation error has taken place, which is repeating a previously learned response when confronted with a new rule. The number of completed categories is also measured in this task (Fig. 2).

\section{Balloon Analogue Risk Task (BART)}

Risky behavior and risky decision-making were assessed with a computerized version of Balloon Analogue Risk Task (Lejuez et al. [56]). Studies have shown that the BART test has an appropriate convergence validity to assess the tendency toward risky behaviors. In this task, a picture of a balloon appeared on the screen, and the participants were asked to pump it by pressing a button. Two boxes were shown, one for temporary earnings and the other for permanent ones. A number on each counter showed the amount of money in it. Every time the participants pumped the balloon, an amount of money was added to the temporary box. The participants had the option of clicking on the "claim money" button instead of pumping further. If they did, their earnings would be transferred to the permanent box, and a new balloon would appear on the screen, restarting the process (the balloons were limited in number to 30). If the balloon popped from pumping, all the money in the temporary box would be lost. Therefore, with each pump, the participants increased the temporary earnings, but also risked losing everything earned in the temporary box. There was no specific breakpoint for the bursting of the balloons; therefore, the participants had to engage in risky decision-making in an uncertain situation. Risk-taking decision-makers tend to ignore the risk of popping the balloons and pump more in order to gain larger amounts of money. In this task, the primary score and the risk-taking indicator is the adjusted average number of pumps for unexploded balloons. The other task scores include the min- imum and maximum number of pumps for each balloon [26] (Fig. 2).

\section{Go/No-Go task}

Response inhibition is an aspect of executive function and is highly correlated with cognitive control [57]. Go/No-Go is a neuropsychological task widely used to assess response inhibition. In this task, a plane appeared in the middle of the screen facing either upward, downward, right, or left. Go and No-Go conditions appeared randomly and in no particular order. In the version used in this study, 100 planes (one plane presented per trial) appeared in the middle of the screen. The participants were instructed to press the button indicating the plane's direction as quickly as they could. For half of the plane stimuli, a "beep" sound was presented as No-Go stimulus, after hearing which the participants had to withhold their response. The number of correct responses to No-Go stimuli shows the subject's ability to inhibit the response. It takes approximately seven minutes to complete this task [26] (Fig. 2).

\section{Desires for Drug Questionnaire (DDQ)}

DDQ consists of 14 items, the original version of which was designed to measure heroin craving [58]. But it was used later to measure craving for other substances. Franken et al. [58] measured the factor structure of this questionnaire and showed that it has good convergent validity, internal consistency, and test-retest reliability. Also, they reported the Cronbach's alpha of this questionnaire equal to 0.85 [58].

\section{Statistical Analysis}

SPSS version 24 (IBM Co., Armonk, NY, USA) was used to analyzethe collected data. Toevaluatedatanormality and homogeneity of variance, we used the Shapiro-Wilk and Levin test. To measure the effectiveness of each treatment and their combined effects on the improvement of executive functions and craving, a mixed model ANOVA was used, with time (pre-test, post-test, follow-up) as the within-subject factor and group (tDCS, MBSAT, tDCS/ MBSAT, sham) as the between-subject factor. Mauchly's test was used to evaluate the sphericity of the data before performing the repeated measures ANOVA. In case that the assumption of sphericity was violated, the degrees of freedom were corrected using Greenhouse-Geisser esti- 
mates of sphericity. Posthoc analysis was done using the Bonferroni correction.

\section{RESULTS}

\section{Data Overview}

In terms of the demographic characteristics of the study youths with methamphetamine dependence, there was no difference between the groups (Table 2). Adolescents in the present study tolerated the electrical stimulation well and did not report significant side effects (Table 3). The data overview including the means and standard deviations of executive functions and craving before, after and 1 month following the intervention is presented in Table 4 and Figure 3.

Table 3. Reported tDCS side effects during stimulation

\begin{tabular}{lcccccc}
\hline \multicolumn{1}{c}{ tDCS session } & Itching sensation & Burning sensation & Pain & Tingling & Fatigue & Trouble concentrating \\
\hline tDCS + MBSAT & 9 & 10 & 6 & 12 & 5 \\
Anodal I-DLPFC & 8 & 8 & 5 & 12 & 6 \\
Sham tDCS & 6 & 5 & 3 & 6 & 2 \\
$\chi^{2}$ (active vs. sham) & 3.39 & 3.20 & 1.57 & 4.43 & 1.36 \\
$p$ value & 0.75 & 0.78 & 0.95 & 0.61 & 0.85 \\
\hline
\end{tabular}

Values are presented as number.

tDCS, transcranial direct current stimulation; MBSAT, mindfulness-based substance abuse treatment; DLPFC, dorsolateral prefrontal cortex.

Table 4. Means and standard deviations of EF tasks and craving before, after and 1 month following intervention

\begin{tabular}{|c|c|c|c|c|c|c|}
\hline \multirow{2}{*}{ Task } & \multirow{2}{*}{$\begin{array}{l}\text { Outcome } \\
\text { measures }\end{array}$} & \multirow{2}{*}{ Time } & \multicolumn{4}{|c|}{ Group } \\
\hline & & & tDCS & MBSAT & $\mathrm{tDCS}+\mathrm{MBSAT}$ & Sham tDCS \\
\hline \multirow[t]{6}{*}{$\mathrm{N}$} & \multirow[t]{3}{*}{ Accuracy } & Pre-intervention & $74.43 \pm 9.52$ & $74.80 \pm 16.98$ & $73.23 \pm 12.92$ & $73.93 \pm 14.34$ \\
\hline & & Post-intervention & $87.25 \pm 8.66$ & $84.66 \pm 6.25$ & $90.70 \pm 5.83$ & $72.31 \pm 13.68$ \\
\hline & & Follow-up & $85.68 \pm 7.53$ & $82.93 \pm 9.61$ & $87.41 \pm 6.24$ & $71.62 \pm 11.88$ \\
\hline & \multirow[t]{3}{*}{ Response time } & Pre-intervention & $153.87 \pm 18.43$ & $155.33 \pm 25.19$ & $154.35 \pm 58.88$ & $154.81 \pm 30.48$ \\
\hline & & Post-intervention & $128.93 \pm 22.27$ & $126.73 \pm 13.16$ & $111.88 \pm 14.73$ & $154.43 \pm 29.68$ \\
\hline & & Follow-up & $120.68 \pm 24.99$ & $126.46 \pm 9.75$ & $113.70 \pm 16.32$ & $154.75 \pm 29.11$ \\
\hline \multirow[t]{6}{*}{ WCST } & \multirow{3}{*}{$\begin{array}{l}\text { Perseverative } \\
\text { errors }\end{array}$} & Pre-intervention & $16.31 \pm 5.77$ & $16.33 \pm 4.99$ & $17.47 \pm 7.02$ & $15.12 \pm 5.56$ \\
\hline & & Post-intervention & $11.75 \pm 1.94$ & $13.33 \pm 3.51$ & $9.29 \pm 2.39$ & $16.18 \pm 1.97$ \\
\hline & & Follow-up & $12.87 \pm 1.92$ & $12.60 \pm 2.13$ & $11.00 \pm 2.93$ & $15.43 \pm 1.41$ \\
\hline & \multirow{3}{*}{$\begin{array}{l}\text { Completed } \\
\text { categories }\end{array}$} & Pre-intervention & $3.00 \pm 0.96$ & $3.06 \pm 0.96$ & $3.17 \pm 0.95$ & $3.18 \pm 1.04$ \\
\hline & & Post-intervention & $3.75 \pm 0.57$ & $3.93 \pm 0.45$ & $4.23 \pm 0.83$ & $3.43 \pm 0.62$ \\
\hline & & Follow-up & $3.75 \pm 1.00$ & $3.73 \pm 0.96$ & $4.20 \pm 0.90$ & $3.37 \pm 0.80$ \\
\hline \multirow[t]{6}{*}{ BART } & \multirow[t]{3}{*}{ Adjusted value } & Pre-intervention & $34.43 \pm 12.29$ & $33.53 \pm 6.02$ & $31.25 \pm 8.15$ & $33.75 \pm 12.81$ \\
\hline & & Post-intervention & $24.43 \pm 6.70$ & $22.26 \pm 4.06$ & $17.88 \pm 4.18$ & $31.68 \pm 10.05$ \\
\hline & & Follow-up & $25.06 \pm 6.35$ & $21.66 \pm 5.13$ & $19.76 \pm 4.58$ & $31.62 \pm 11.18$ \\
\hline & \multirow[t]{3}{*}{ Max pumping } & Pre-intervention & $60.25 \pm 13.08$ & $58.26 \pm 10.93$ & $59.64 \pm 16.45$ & $57.62 \pm 10.95$ \\
\hline & & Post-intervention & $35.31 \pm 7.49$ & $37.13 \pm 5.56$ & $29.76 \pm 7.71$ & $52.81 \pm 9.04$ \\
\hline & & Follow-up & $40.93 \pm 11.87$ & $41.06 \pm 7.61$ & $32.00 \pm 9.71$ & $48.43 \pm 8.75$ \\
\hline \multirow[t]{6}{*}{ Go/No-Go } & \multirow[t]{3}{*}{ Accuracy Go } & Pre-intervention & $94.75 \pm 6.86$ & $93.13 \pm 9.37$ & $94.76 \pm 4.90$ & $95.18 \pm 6.56$ \\
\hline & & Post-intervention & $96.56 \pm 4.08$ & $97.33 \pm 3.97$ & $98.58 \pm 2.76$ & $94.12 \pm 5.41$ \\
\hline & & Follow-up & $97.06 \pm 2.35$ & $97.46 \pm 2.26$ & $98.00 \pm 2.44$ & $95.13 \pm 5.30$ \\
\hline & \multirow{3}{*}{$\begin{array}{c}\text { Accuracy } \\
\text { No-Go }\end{array}$} & Pre-intervention & $75.62 \pm 14.77$ & $76.26 \pm 13.41$ & $76.37 \pm 12.02$ & $76.37 \pm 12.02$ \\
\hline & & Post-intervention & $82.18 \pm 7.54$ & $83.66 \pm 8.05$ & $85.88 \pm 7.44$ & $76.88 \pm 7.53$ \\
\hline & & Follow-up & $79.25 \pm 7.54$ & $77.80 \pm 4.58$ & $85.94 \pm 5.74$ & $76.31 \pm 5.74$ \\
\hline \multirow[t]{3}{*}{ Craving } & \multirow[t]{3}{*}{ DDQ } & Pre-intervention & $52.25 \pm 11.06$ & $49.46 \pm 5.51$ & $50.29 \pm 13.99$ & $49.56 \pm 6.89$ \\
\hline & & Post-intervention & $43.75 \pm 9.82$ & $36.53 \pm 7.41$ & $31.47 \pm 8.88$ & $47.25 \pm 6.13$ \\
\hline & & Follow-up & $42.81 \pm 8.78$ & $37.00 \pm 7.98$ & $33.82 \pm 10.06$ & $47.37 \pm 5.73$ \\
\hline
\end{tabular}

Values are presented as mean \pm standard deviation.

EF, executive functions; tDCS, transcranial direct current stimulation; MBSAT, mindfulness-based substance abuse treatment; WCST, Wisconsin Card Sorting Test; BART, Balloon Analouge Risk Task; DDQ, Desires for Drug Questionnaire. 


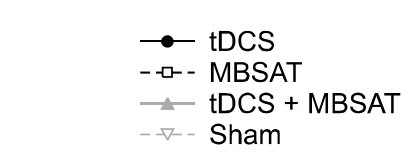

$\mathrm{N}$ back

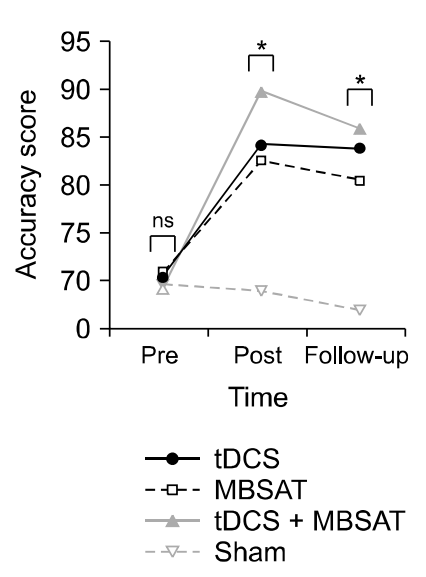

BART
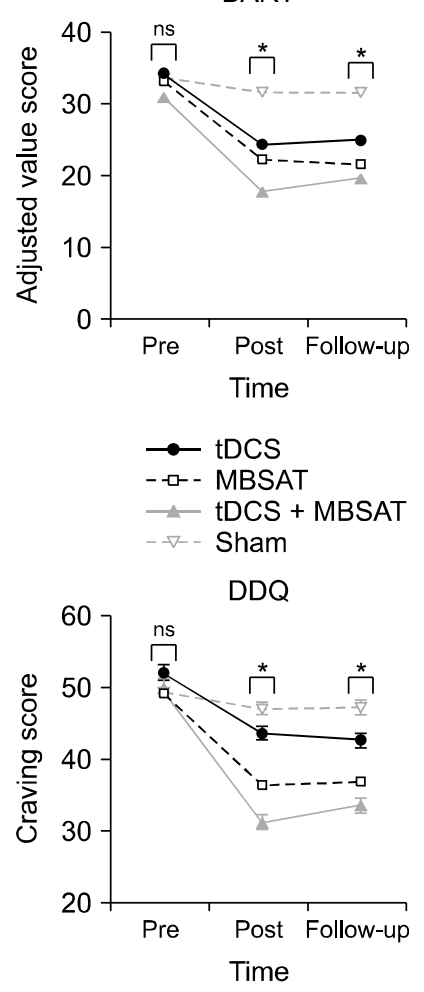

\section{The Effect of tDCS, MBSAT and Combine these Two Methods (tDCS/MBSAT) on Executive Functions and Craving}

To analyze the effectiveness of each intervention (i.e., tDCS, MBSAT, and tDCS/MBSAT) in improving the executive functions and craving, the mixed factorial ANOVA method was used.We refer to Figure 3 to illustrate the pattern of the results of each dependent variable. Results are
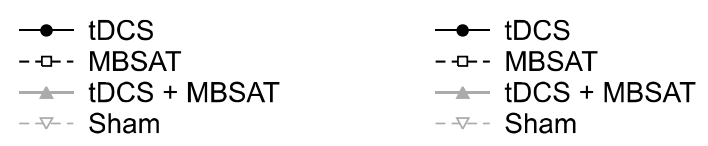

WCST
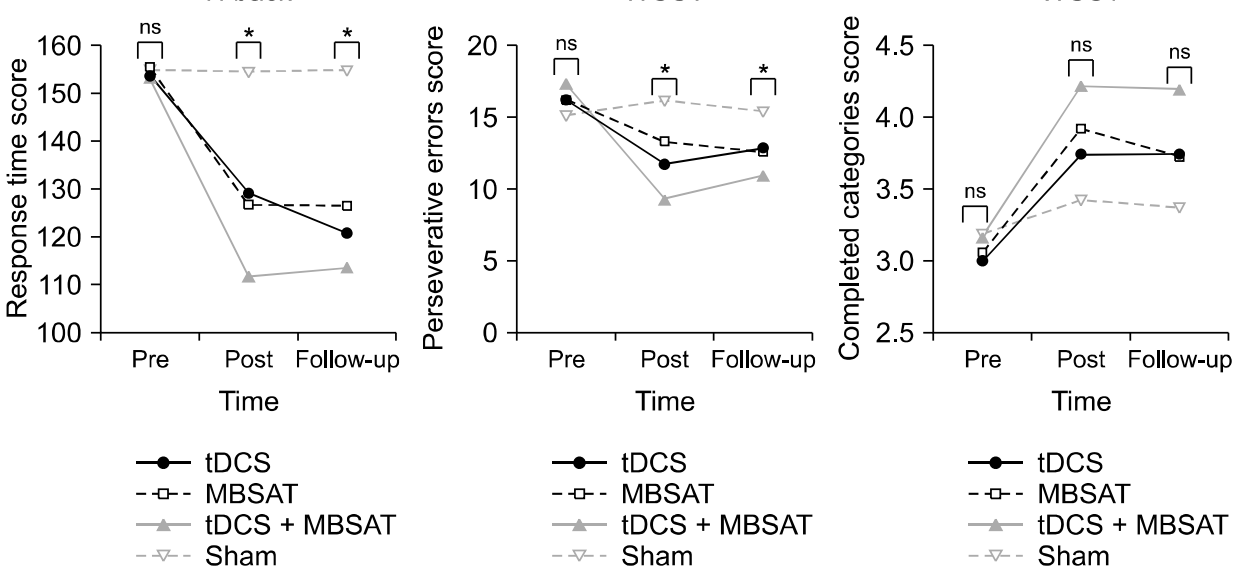

GO/No-Go
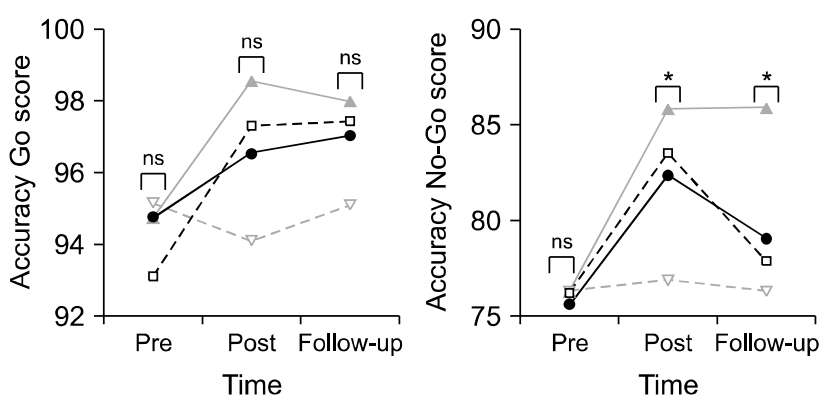

Fig. 3. Performance in the EF tasks (N-back, WCST, BART and Go/ No-GO) and craving before the intervention, after the intervention and 1-month following intervention in research groups (tDCS, MBSAT, PIN-CODES, and sham). tDCS: real tDCS group, sham: sham tDCS group.

tDCS, transcranial direct current stimulation; PIN-CODES, psychological interventions combined with direct electrical stimulation; MBSAT, mindfulness-based substance abuse treatment; WCST, Wisconsin Card Sorting Test; BART, Balloon Analogue Risk Task; DDQ, Desires for Drug Questionnaire.

summarized in Table 5.

For the N-back task, results showed a significant interaction effect of group $\times$ time on both component accuracy $\left(\mathrm{F}_{(6,120)}=8.59, p<0.001, \eta p^{2}=0.30\right)$ and response time $\left(\mathrm{F}_{(6,120)}=8.60, p<0.002, \eta p^{2}=0.20\right)$. As well as a significant main effect of group on accuracy $\left(\mathrm{F}_{(3,60)}=8.59, p<\right.$ $\left.0.020, \eta p^{2}=0.20\right)$ and response time $\left(\mathrm{F}_{(3,60)}=5.44, p<\right.$ $\left.0.002, \eta p^{2}=0.21\right)$. The between-group post hoc comparisons 
Table 5. Results of mixed-model ANOVAs for the effects of group (tDCS, MBSAT, PIN-CODES and Sham) and time (pre-intervention, post-intervention, follow-up) on executive functions and craving

\begin{tabular}{|c|c|c|c|c|c|c|c|}
\hline Task & $\begin{array}{l}\text { Outcome } \\
\text { measures }\end{array}$ & Source & df & f & $p$ value & $\eta p^{2}$ & Pairwise comparisons (Bonferroni) \\
\hline \multirow[t]{6}{*}{$N$} & \multirow[t]{3}{*}{ Accuracy } & Time & 2.120 & 44.53 & $0.001^{*}$ & 0.42 & \multirow{3}{*}{$\begin{array}{c}\mathrm{tDCS}+\mathrm{MBSAT}>\text { Sham }(p<0.009) \\
\mathrm{tDCS}>\text { Sham }(p<0.031)\end{array}$} \\
\hline & & Group & 3.60 & 8.60 & $0.020^{*}$ & 0.30 & \\
\hline & & Time $\times$ group & 6.120 & 8.59 & $0.001 *$ & 0.30 & \\
\hline & \multirow[t]{3}{*}{ Response time } & Time & 2.120 & 39.64 & $0.001 *$ & 0.39 & \multirow[t]{3}{*}{$\mathrm{tDCS}+\mathrm{MBSAT}>\operatorname{Sham}(p<0.006)$} \\
\hline & & Group & 3.60 & 5.44 & $0.002 *$ & 0.21 & \\
\hline & & Time $\times$ group & 6.120 & 5.22 & $0.002 *$ & 0.20 & \\
\hline \multirow[t]{6}{*}{ WCST } & \multirow{3}{*}{$\begin{array}{l}\text { Perseverative } \\
\text { errors }\end{array}$} & Time & 2.120 & 18.21 & $0.001 *$ & 0.23 & \multirow{6}{*}{ tDCS + MBSAT $>$ Sham $(p<0.004)$} \\
\hline & & Group & 3.60 & 3.43 & $0.022^{*}$ & 0.14 & \\
\hline & & Time $\times$ group & 6.120 & 4.55 & $0.001 *$ & 0.18 & \\
\hline & \multirow{3}{*}{$\begin{array}{l}\text { Completed } \\
\text { categories }\end{array}$} & Time & 2.120 & 18.84 & $0.001 *$ & 0.23 & \\
\hline & & Group & 3.60 & 0.449 & 0.719 & 0.10 & \\
\hline & & Time $\times$ group & 6.120 & 0.319 & 0.926 & 0.01 & \\
\hline \multirow[t]{6}{*}{ BART } & \multirow[t]{3}{*}{ Adjusted value } & Time & 2.120 & 44.15 & $0.001 *$ & 0.42 & \multirow{3}{*}{$\begin{array}{l}\text { tDCS }+ \text { MBSAT }>\text { Sham }(p<0.001) \\
\text { MBSAT }>\text { Sham }(p<0.041)\end{array}$} \\
\hline & & Group & 3.60 & 3.99 & $0.012^{*}$ & 0.16 & \\
\hline & & Time $\times$ group & 6.120 & 3.19 & $0.013^{*}$ & 0.13 & \\
\hline & \multirow[t]{3}{*}{ Max pumping } & Time & 2.120 & 96.75 & $0.001^{*}$ & 0.61 & \multirow{6}{*}{$\begin{array}{l}\mathrm{tDCS}+\text { MBSAT }>\text { Sham }(p<0.001) \\
\text { tDCS }>\text { Sham }(p<0.005) ; \text { MBSAT } \\
>\text { Sham }(p<0.006)\end{array}$} \\
\hline & & Group & 3.60 & 6.05 & $0.006^{*}$ & 0.18 & \\
\hline & & Time $\times$ group & 6.120 & 6.42 & $0.001 *$ & 0.24 & \\
\hline \multirow[t]{6}{*}{ Go/No-Go } & \multirow[t]{3}{*}{ Accuracy Go } & Time & 2.120 & 5.29 & $0.009 *$ & 0.08 & \\
\hline & & Group & 3.60 & 1.25 & 0.296 & 0.05 & \\
\hline & & Time $\times$ group & 6.120 & 1.12 & 0.35 & 0.05 & \\
\hline & Accuracy & Time & 2.120 & 8.63 & $0.001 *$ & 0.12 & \multirow[t]{3}{*}{$\mathrm{tDCS}+\mathrm{MBSAT}>$ Sham $(p<0.047)$} \\
\hline & No-Go & Group & 3.60 & 3.19 & $0.047^{*}$ & 0.12 & \\
\hline & & Time $\times$ group & 6.120 & 1.79 & 0.140 & 0.08 & \\
\hline \multirow[t]{3}{*}{ Craving } & \multirow[t]{3}{*}{ DDQ } & Time & 2.120 & 117.44 & $0.001 *$ & 0.66 & \multirow{3}{*}{$\begin{array}{l}\text { tDCS }+ \text { MBSAT }>\text { Sham }(p<0.008) \\
\text { MBSAT }>\text { Sham }(p<0.032) ; \text { tDCS } \\
>\text { Sham }(p<0.041)\end{array}$} \\
\hline & & Group & 3.60 & 4.86 & $0.004^{*}$ & & \\
\hline & & Time $\times$ group & 6.120 & 12.11 & $0.001 *$ & 0.37 & \\
\hline
\end{tabular}

tDCS, transcranial direct current stimulation; PIN-CODES, psychological interventions combined with direct electrical stimulation; MBSAT, mindfulness-based substance abuse treatment; WCST, Wisconsin Card Sorting Test; BART, Balloon Analog Risk Task; DDQ, Desires for Drug Questionnaire.

Significant results are highlighted $(p<0.05)$ in asterisk; Note: Only significant pairwise comparisons are shown.

showed no significant difference in the pre-intervention measurement $\left(F_{\text {Accuracy }}=0.04, p=0.989 ; F_{\text {Response Time }}=\right.$ $0.004, p=0.997)$, but significant between-group differences in the post-intervention $\left(F_{\text {Accuracy }}=12.42, p=0.001\right.$; $\left.F_{\text {Response Time }}=11.52, p=0.001\right)$ and follow-up intervention $\left(F_{\text {Accuracy }}=10.02, p=0.001 ; F_{\text {Response Time }}=11.40\right.$, $p=0.001)$ measurements. Thepaired samples post-hoc $t$ tests showed anincrease and decreaseofaccuracy and response timein the post-intervention (Accuracy: tDCS/ MBSAT: $t=6.87, p<0.001$, tDCS: $t=6.63, p<0.001$, MBSAT: $t=2.92, p<0.011$. Response Time: tDCS/ MBSAT: $t=-3.44, p<0.003$, tDCS: $t=-5.44, p<$ 0.001$, MBSAT: $t=-3.87, p<0.002)$ and follow-up (Accuracy: $\mathrm{tDCS} / \mathrm{MBSAT}: t=5.52, p<0.001$, tDCS: $t=$ 4.73, $p<0.001$, MBSAT: $t=2.72, p<0.017$. Response Time: $\mathrm{tDCS} / \mathrm{MBSAT}: t=-3.32, p<0.004, \mathrm{tDCS}: t=-$
6.83, $p<0.001$, MBSAT: $t=-4.22, p<0.001)$ measurements compared to the pre-intervention measurement in the each intervention,but not sham stimulation group. All other comparisons did not reach significance, $p>$ 0.05 .

For the WCST task, results showed a significant interaction effect of group $\times$ time on perseverative errors component $\left(\mathrm{F}_{(6,120)}=4.55, p<0.001, \eta p^{2}=0.18\right)$ and no significant interaction effect of group $\times$ time on completed categories component $\left(\mathrm{F}_{(6,120)}=0.319, p<0.926, \eta p^{2}=\right.$ 0.01). Also results showed a significant main effect of group on perseverative errors $\left(\mathrm{F}_{(3,60)}=3.43, p<0.022\right.$, $\left.\eta p^{2}=0.14\right)$ but no significant main effect of group on completed categories $\left(\mathrm{F}_{(3,60)}=0.44, p<0.719, \eta p^{2}=\right.$ $0.10)$. The between-group comparisons showed no significant difference in the pre-intervention measurement 
$\left(\digamma_{\text {Perseverative Errors }}=0.431, p=0.731 ; F_{\text {Completed Categories }}=\right.$ $0.136, p=0.937)$. In perseverative errors asignificant between-group differences in the post-intervention $\left(F_{\text {Perseverative Errors }}=21.63, p=0.001\right)$ and follow-up intervention measurements $\left(F_{\text {Perseverative Errors }}=11.52, p=0.001\right)$ but in the Completed Categories no significant difference in the post-intervention measurement $\left(F_{\text {Completed Categories }}=\right.$ $1.60, p=0.198)$ and follow-up intervention $\left(F_{\text {Completed Categories }}=\right.$ 1.03, $p=0.383$ ) measurements.

The post-hoc $t$ tests analysis showed a significant decrease and increase of WCST perseverative errors and completed categories, respectively, between pre-intervention and post-interventionmeasurements (Perseverative Errors: tDCS/MBSAT: $t=-5.01, p<0.001$, tDCS: $t=-2.98$, $p<0.009$, MBSAT: $t=-1.78, p>0.096$. Completed Categories: tDCS/MBSAT: $t=4.51, p<0.001$, tDCS: $t=$ 1.46, $p<0.018$, MBSAT: $t=1.45, p<0.007)$ and pre-intervention vs. follow-up measurements (Perseverative Errors: $\mathrm{tDCS} / \mathrm{MBSAT}: t=-3.69, p<0.002$, tDCS: $t=-2.18$, $p<0.045$, MBSAT: $t=-3.32, p<0.005$. Completed Categories: tDCS/MBSAT: $t=4.01, p<0.001$, tDCS: $t=$ 2.08, $p<0.050$, MBSAT: $t=2.46, p<0.027)$ in each intervention, but not sham stimulation group. All other comparisons did not reach significance, $p>0.05$.

For the BART task, results showed a significant interaction effect of group $\times$ time on both Adjusted value $\left(\mathrm{F}_{(6,120)}=\right.$ 3.19, $\left.p<0.013, \eta p^{2}=0.13\right)$ and Max pumping $\left(\mathrm{F}_{(6,120)}=\right.$ 6.42, $\left.p<0.001, \eta p^{2}=0.24\right)$. As well as the results showed that a significant main effect of group on Adjusted value $\left(\mathrm{F}_{(3,60)}=3.99, p<0.012, \eta p^{2}=0.16\right)$ and Max pumping $\left(\mathrm{F}_{(3,60)}=6.05, p<0.006, \eta p^{2}=0.18\right)$. The between-group comparisons showed no significant difference in the pre-intervention measurement $\left(F_{\text {Adjusted value }}=\right.$ $\left.0.307, p=0.821 ; F_{\text {Max Pumping }}=0.136, p=0.938\right)$, but significant between-group differences in the post-intervention $\left(F_{\text {Adjusted value }}=12.06, p=0.001 ; F_{\text {Max Pumping }}=\right.$ $27.54, p=0.001)$ and follow-up intervention $\left(F_{\text {Adjusted value }}=\right.$ 8.27, $\left.p=0.001 ; F_{\text {Max Pumping }}=8.28, p=0.001\right)$ measurements. Also, the post-hoct tests analysis showed a significant decrease of BART Adjusted value and Max pumping, respectively, between pre-intervention and post-intervention measurements (Adjusted value: $\mathrm{tDCS} / \mathrm{MBSAT}: t=$ $-7.30, p<0.001$, tDCS: $t=-2.75, p<0.015$, MBSAT: $t=-4.90, p<0.001$. Max pumping: tDCS/MBSAT: $t=$ $-6.90, p<0.001$, tDCS: $t=-7.36, p<0.001$, MBSAT: $t=-6.37, p<0.001)$ and pre-intervention vs. follow-up measurements (Adjusted value: tDCS/MBSAT: $t=-7.57$, $p<0.001$, tDCS: $t=-2.98, p<0.009$, MBSAT: $t=-5.38$, $p<0.001$. Max pumping: tDCS/MBSAT: $t=-7.02, p<$ 0.001 , tDCS: $t=-4.50, p<0.001$, MBSAT: $t=-7.29, p<$ 0.001 ) in the each intervention,but not sham stimulation group. All other comparisons did not reach significance, $p>$ 0.05 .

For the Go/No-Go task, results showed no significant interaction effect of group $\times$ time on both Accuracy Go $\left(\mathrm{F}_{(6,120)}=1.12, p<0.35, \eta p^{2}=0.05\right)$ and Accuracy No-Go $\left(\mathrm{F}_{(6,120)}=1.79, p<0.14, \eta p^{2}=0.08\right)$. As well as the results showed that a no significant main effect of group on Accuracy Go $\left(\mathrm{F}_{(3,60)}=1.25, p<0.296, \eta p^{2}=\right.$ 0.05 ) but in Accuracy No-Go result showed a significant main effect of group $\left(\mathrm{F}_{(3,60)}=3.19, p<0.047, \eta p^{2}=0.12\right)$. The between-group comparisons showed no significant difference in the pre-intervention measurement $\left(F_{\text {Accuracy Go }}=\right.$ $\left.0.254, p=0.858 ; F_{\text {Accuracy No-Go }}=0.016, p=0.997\right)$. In Accuracy No-Go a significant between-group differences in the post-intervention $\left(F_{\text {Accuracy No-Go }}=5.73, p=0.002\right)$ and follow-up intervention measurements $\left(F_{\text {Accuracy No-Go }}=\right.$ $6.71, p=0.001)$ but in the Accuracy Go no significant difference in the post-intervention measurement $\left(F_{\text {Accuracy Go }}=\right.$ 2.34, $p=0.068)$ and follow-up intervention $\left(F_{\text {Accuracy Go }}=\right.$ 1.95, $p=0.130)$ measurements. The post-hoc $t$ tests showed an increase and decrease of Accuracy Go and Accuracy No-Go, respectively, between pre-intervention and post-intervention measurements (Accuracy Go: Pin-Codes: $t=3.80, p<0.002$. Accuracy No-Go: tDCS/ MBSAT: $t=3.37, p<0.004)$ and pre-intervention vs. follow-up measurements (Accuracy Go: tDCS/MBSAT: $t=$ 2.97, $p<0.009$. Accuracy No-Go: Pin-Codes: $t=3.18, p<$ 0.006) in the tDCS + MBSAT intervention, but not other intervention and sham stimulation group. All other comparisons did not reach significance, $p>0.05$.

Finally, the DDQ scores showed a significant interaction effect of group $\times$ time on craving $\left(\mathrm{F}_{(6,120)}=12.11, p<\right.$ $\left.0.001, \eta p^{2}=0.37\right)$. Also results showed that a significant main effect of group on craving $\left(\mathrm{F}_{(3,60)}=3.99, p<0.012\right.$, $\left.\eta p^{2}=0.16\right)$. The between-group comparisons showed no significant difference in the pre-intervention measurement $(F=0.259, p=0.854)$, but significant betweengroup differences in the post-intervention $(\mathrm{F}=16.51, p=$ $0.001)$ and follow-up intervention ( $\mathrm{F}=11.13, p=0.001)$ measurements. The post-hoc $t$ tests showed a decrease of craving, respectively, between pre-intervention and post- 
Table 6. Correlation between craving reduction rate after the intervention with rates of changes from pre- to post-intervention in the EFs tasks

\begin{tabular}{llllllllrr}
\hline & Group & WM accuracy & WM RT & BART-AV & BART-Max & WCST-PE & WCST-CC & $\begin{array}{c}\text { Accuracy } \\
\text { Go }\end{array}$ & $\begin{array}{c}\text { Accuracy } \\
\text { No-Go }\end{array}$ \\
\hline \multirow{2}{*}{ tDCS + MBSAT } & Pearson coeffitient & -0.625 & 0.442 & 0.700 & 0.551 & 0.485 & -0.171 & -0.134 & -0.103 \\
& $p$ value (2-taild) & $0.007^{* * * * * *}$ & 0.075 & $0.002^{* * * * *}$ & $0.022^{* * * * *}$ & $0.049^{* * * * *}$ & 0.512 & 0.609 & 0.693 \\
\multirow{2}{*}{ tDCS } & Pearson coeffitient & -0.438 & 0.380 & 0.569 & 0.353 & 0.332 & -0.339 & -0.031 & -0.297 \\
& $p$ value (2-taild) & 0.090 & 0.146 & $0.021^{* * * * *}$ & 0.180 & 0.209 & 0.199 & 0.910 & 0.264 \\
\multirow{2}{*}{ MBSAT } & Pearson coeffitient & -0.416 & 0.204 & 0.543 & 0.400 & 0.384 & -0.072 & -0.212 & -0.101 \\
& $p$ value (2-taild) & 0.123 & 0.466 & $0.036^{* * *}$ & 0.140 & 0.158 & 0.800 & 0.449 & 0.720 \\
Sham & Pearson coeffitient & -0.145 & 0.187 & 0.021 & -0.170 & 0.161 & -0.245 & -0.372 & -0.160 \\
& $p$ value (2-taild) & 0.592 & 0.489 & 0.939 & 0.529 & 0.551 & 0.323 & 0.156 & 0.554 \\
\hline
\end{tabular}

EF, executive functions; WM accuracy, working memory accuracy; WM RT, working memory response time; WCST-PE, perseverative errors in the Wisconsin Card Sorting Task; WCST-CC, completed categories in the Wisconsin Card Sorting Task; BART-AV, adjusted value in the Balloon Analogue Risk Task; BART-Max, maximum number of pumps in the Balloon Analogue Risk Task.

Significant results are highlighted in $\left(^{* * *}\right)$ at 0.01 level $\left({ }^{* *}\right)$ and 0.05 level $\left({ }^{*}\right)$.

intervention measurements (tDCS/MBSAT: $t=-8.82, p<$ 0.001 , tDCS: $t=-6.82, p<0.001$, MBSAT: $t=-8.61, p<$ $0.001)$ and pre-intervention vs. follow-up measurements (tDCS/MBSAT: $t=-8.02, p<0.001$, tDCS: $t=-6.36, p<$ 0.001, MBSAT: $t=-7.44, p<0.001$ ) in each intervention,but not sham stimulation group. All other comparisons did not reach significance, $p>0.05$.

Also, to investigatewhether percentage of changes in cravingwere correlated with EF task performance, Pearson's correlationsshowed a significant correlation between craving and the following EF measures in the tDCS + MBSAT intervention (WM accuracy $(p<0.007)$, BARTAV $(p<$ 0.002), BART Max $(p<0.022)$, WCST PE $(p<0.049)$, but not other intervention and sham stimulation group (Table 6). This correlation indicates that the reducting in craving is associated with the increase in EFs, specifically in the $\mathrm{t} 2$ condition.

\section{DISCUSSION}

More than a decade of research has shown the potential of tDCS and MBIs in the treatment of SUD. Yet, new studies indicate that tDCS would be more effective if used as a complementary technique, to enhance the excitability of a neuronal network and hence increase the effects of a psychological intervention [37-41], namely MBSAT as investigated in our clinical trial.

In the current preregistered clinical trial, a randomized, single-blind design was used to examine the effects of the combination of bifrontal tDCS with MBSAT on EFs and craving in early abstinent youths with methamphetamine use disorder. Our study showed that the combination therapy significantly reduced craving and improved executive functionsincluding working memory, risk-taking behavior, inhibitory control and cognitive flexibility compared to other treatment groups.

Our results are in line with recent advances and showed that tDCS + MBSAT is more beneficial for improving EFs and craving in youths with methamphetamine use disorders (as compared to each intervention as a monotherapy and as compared to sham), suggesting a new treatment approach in the treatment of drug addiction. The effect of tDCS depends on the activity of the brain during stimulation [59] and according to the activity-selectivity hypothesis, when tDCS is given to an activated neural network, it results in increased neuroplasticity [60]. So, when a neural network is modified by psychological intervention, this sets the stage for a larger potential for efficacy of tDCS [61]. Therefore, the combination of tDCS method with psychological interventions leads to an increase in the effectiveness of this treatment method and significantly increases the clinical effects [50].

Previous studies show that in both the clinical $[28,29,62]$ and healthy populations [26,27], EFs are enhanced by tDCS. Given thatthese EFs are also targeted by mindfulness, the greater reduction of craving in the tDCS + MBSAT group may be related to the direct involvement of enhancing executive functions. Also, the results of our research showed that there is a significant correlation between changes in executive functions and cravings in the combined group (tDCS + MBSAT). From a neurobiological point of view, the areas of the brain involved in cravings for drugs are so extensive that they include complex fron- 
tal cortical and subcortical circuits such as DLPFC, anterior cingulate and orbitofrontal cortex that is closely related to insular functions [63]. Functional imaging studies show that there is a positive correlation between cravings with insular activity [64] and modulation of insular activity after stimulation of the DLPFC area can be an explanation for the decrease in cravings. Therefore, the additional effects of the combined approach (tDCS + MBSAT) can affect different areas of the frontal cortex and lead to a wide reduction in craving. Recent studies from our group indicated that anodal tDCS over the left DLPFC decrease craving [65] and improves frontal executive functions in severe alcoholics [66]. Fernández-Serrano et al. [67] showed that alcohol and polysubstance abusers have clinically significant deficits in executive functions and decision-making. Studies in the field of neuroscience addiction have determined a link between substance use and cognitive deficits. Spronk et al. [68] indicated that long-term cocaine use is related with cognitive destruction in most cognitive components, with firm evidence on executive functions such as decision-making,working memory, sustained attention, and response inhibition. Persistent deficits in executive functions have also been shown inusers of opiates, methamphetamine or alcohol [69].

Therapeutic effects of MBIs on drug use and relapse prevention may be explained by the effects of mindfulness training on neurocognitive mechanisms involved in substance use disorder. The MBls work as mental training programs to boost neurocognitive deficits that were developed during the course of the addiction [44]. Promotion of increased EFs through mindfulness training could thus possibly enable the individual to achieve self-control over automatic substance use behavior. This beneficial process is suggested to enhance functional connectivity between prefrontal control areas and striatal brain circuitry engaged in reward processing and habitual responses [44]. Modified communication in this frontostriatal circuit may determine how MBls can restructure reward processes snatched during the addictive process [70]. Therefore, MBIs, as powerful neurocognitive resources, can be used to adjust the automaticity, hedonic dysregulation, and stress reactivity that undergirds SUDs and relapse. This evidence supportsthe idea that mindfulness training may reduce substance use behavior by enhancing activation in the prefrontal-mediated cognitive control networks and, inso doing, facilitate the regulation of reactivity to substance cues [71].

In sum, our results provide evidence for the promising clinical application of combined MBI-tDCS in drug addiction, and suggest a combined protocol (tDCS with mindfulness) in the treatment of people with substance use disorders. Future studies are recommended to investigate the effects of tDCS in combination with other psychological interventions such as cognitive-behavioral treatments. Despite promising avenues for the treatment of drug abuse by using a combination of tDCS and mindfulness intervention,some limitations need to be considered when interpreting our findings. The follow-up stage in this study lasted one Month, so, to evaluate the long-term effects of treatment, it is recommended that studies with longer follow-up periods be conducted. Another limitation of the present study was the lack of a larger number of research samples. Future research should replicate our interventions in larger samples sizes. Another limitation of the present study was the lack of a tool for measuring brain changes after intervention, which is suggested by future research to use brain imaging techniques to better describe brain changes.

\section{Acknowledgments}

We express our sincere gratitude to the Welfare Organization of Ardabil Province for providing the research samples and the Research Center for Behavioral Cognitive Neurosciences for the provision of research tools.

\section{Conflicts of Interest}

No potential conflict of interest relevant to this article was reported.

\section{Author Contributions}

Investigation, Data curation, Formal analysis, Data collection, Visualization, Validation, Writing-Original Draft: Jaber Alizadehgoradel. Conceptualization, Methodology, Software: Saeed Imani, Vahid Nejati. Supervision, Writing Review \& Editing: Marie-Anne Vanderhasselt, Behnam Molaei, and Mohammad Ali Salehinejad. Investigation, Data collection: Shirin Ahmadi and Mina Taherifard.

\section{ORCID}

Jaber Alizadehgoradel 
https://orcid.org/0000-0003-4803-0607

Saeed Imani https://orcid.org/0000-0001-5290-2995

Vahid Nejati

https://orcid.org/0000-0002-5933-4422

Marie-Anne Vanderhasselt

https://orcid.org/0000-0002-4045-1055

Behnam Molaei https://orcid.org/0000-0002-9859-6521

Mohammad Ali Salehinejad

https://orcid.org/0000-0003-1913-4677

Shirin Ahmadi

https://orcid.org/0000-0003-0189-9575

Mina Taherifard https://orcid.org/0000-0002-5687-794X

\section{REFERENCES}

1. Bari A, DiCesare J, Babayan D, Runcie M, Sparks H, Wilson B. Neuromodulation for substance addiction in human subjects: a review. Neurosci Biobehav Rev 2018;95:33-43.

2. Johnston LD, O'Malley PM, Bachman JG, Schulenberg JE, Miech RA. Monitoring the Future national survey results on drug use, 1975-2015: volume II, college students and adults ages 19-55. Ann Arbor:Institute for Social Research, The University of Michigan;2016.

3. Volkow ND, Koob GF, McLellan AT. Neurobiologic advances from the brain disease model of addiction. NEng/JMed 2016; 374:363-371.

4. Domínguez-Salas S, Díaz-Batanero C, Lozano-Rojas OM, Verdejo-García A. Impact of general cognition and executive function deficits on addiction treatment outcomes: systematic review and discussion of neurocognitive pathways. Neurosci Biobehav Rev 2016;71:772-801.

5. Pentz MA, Spruijt-Metz D, Chou CP, Riggs NR. High calorie, low nutrient food/beverage intake and video gaming in children as potential signals for addictive behavior. Int I Environ Res Public Health 2011;8:4406-4424.

6. Riggs NR, Spruijt-Metz D, Chou CP, Pentz MA. Relationships between executive cognitive function and lifetime substance use and obesity-related behaviors in fourth grade youth. Child Neuropsychol 2012;18:1-11.

7. Dwyer JB, McQuown SC, Leslie FM. The dynamic effects of nicotine on the developing brain. Pharmacol Ther 2009;122: 125-139.

8. Kandel ER, Kandel DB. Shattuck Lecture. A molecular basis for nicotine as a gateway drug. N Eng/ J Med 2014;371:932 943.

9. Pentz MA, Shin H, Riggs N, Unger JB, Collison KL, Chou CP. Parent, peer, and executive function relationships to early adolescent e-cigarette use: a substance use pathway? Addict Behav 2015:42:73-78.

10. Stevens L, Verdejo-García A, Goudriaan AE, Roeyers H, Dom $\mathrm{G}$, Vanderplasschen W. Impulsivity as a vulnerability factor for poor addiction treatment outcomes: a review of neurocognitive findings among individuals with substance use disorders. J Subst Abuse Treat 2014;47:58-72.
11. Kalivas PW, O'Brien C. Drug addiction as a pathology of staged neuroplasticity. Neuropsychopharmacology 2008;33:166-180.

12. Hartwell KJ, Johnson KA, Li X, Myrick H, LeMatty T, George MS, et al. Neural correlates of craving and resisting craving for tobacco in nicotine dependent smokers. Addict Biol 2011;16. 654-666.

13. Sarkis RA, Kaur N, Camprodon JA. Transcranial direct current stimulation (tDCS): modulation of executive function in health and disease. Curr Behav Neurosci Rep 2014;1:74-85.

14. Lee JC, Kenney-Jung DL, Blacker CJ, Doruk Camsari D, Lewis CP. Transcranial direct current stimulation in child and adolescent psychiatric disorders. Child Adolesc Psychiatr Clin N Am 2019;28:61-78.

15. Nitsche MA, Fricke K, Henschke U, Schlitterlau A, Liebetanz D, Lang N, et al. Pharmacological modulation of cortical excitability shifts induced by transcranial direct current stimulation in humans. J Physiol 2003;553(Pt 1):293-301.

16. Salehinejad MA, Nejati V, Mosayebi-Samani M, Mohammadi A, Wischnewski M, Kuo MF, et al. Transcranial direct current stimulation in ADHD: a systematic review of efficacy, safety, and protocol-induced electrical field modeling results. Neurosci Bull 2020;36:1191-1212.

17. Osório AAC, Brunoni AR. Transcranial direct current stimulation in children with autism spectrum disorder: a systematic scoping review. Dev Med Child Neurol 2019;61:298-304.

18. Muszkat D, Polanczyk GV, Dias TG, Brunoni AR. Transcranial direct current stimulation in child and adolescent psychiatry. J Child Adolesc Psychopharmacol 2016;26:590-597.

19. Rivera-Urbina GN, Nitsche MA, Vicario CM, Molero-Chamizo A. Applications of transcranial direct current stimulation in children and pediatrics. Rev Neurosci 2017;28:173-184.

20. Salehinejad MA, Wischnewski M, Nejati V, Vicario CM, Nitsche MA. Correction: Transcranial direct current stimulation in attention-deficit hyperactivity disorder: a meta-analysis of neuropsychological deficits. PLoS One 2019;14:e0221613.

21. Coles AS, Kozak K, George TP. A review of brain stimulation methods to treat substance use disorders. Am J Addict 2018; 27:71-91.

22. Holla B, Biswal J, Ramesh V, Shivakumar V, Bharath RD, Benegal $\mathrm{V}$, et al. Effect of prefrontal tDCS on resting brain $\mathrm{FMR} /$ graph measures in Alcohol Use Disorders: a randomized, double-blind, sham-controlled study. Prog Neuropsychopharmacol Biol Psychiatry 2020;102:109950.

23. Vanderhasselt MA, Allaert J, De Raedt R, Baeken C, Krebs RM, Herremans S. Bifrontal tDCS applied to the dorsolateral prefrontal cortex in heavy drinkers: influence on reward-triggered approach bias and alcohol consumption. Brain Cogn 2020; 138:105512.

24. Ahn YH, Sohn HJ, Park JS, Ahn TG, Shin YB, Park M, et al. Effect of bihemispheric anodal transcranial direct current stimulation for dysphagia in chronic stroke patients: a randomized clinical trial. J Rehabil Med 2017;49:30-35.

25. Salehinejad MA, Ghayerin E, Nejati V, Yavari F, Nitsche MA. 
Domain-specific involvement of the right posterior parietal cortex in attention network and attentional control of ADHD: a randomized, cross-over, sham-controlled tDCS study. Neuroscience 2020;444:149-159.

26. Nejati $\mathrm{V}$, Salehinejad MA, Nitsche MA. Interaction of the left dorsolateral prefrontal cortex (I-DLPFC) and right orbitofrontal cortex (OFC) in hot and cold executive functions: evidence from transcranial direct current stimulation (tDCS). Neuroscience 2018;369:109-123.

27. Ghanavati E, Salehinejad MA, Nejati V, Nitsche MA. Differential role of prefrontal, temporal and parietal cortices in verbal and figural fluency: implications for the supramodal contribution of executive functions. Sci Rep 2019;9:3700.

28. Molavi P, Aziziaram S, Basharpoor S, Atadokht A, Nitsche MA, Salehinejad MA. Repeated transcranial direct current stimulation of dorsolateral-prefrontal cortex improves executive functions, cognitive reappraisal emotion regulation, and control over emotional processing in borderline personality disorder: a randomized, sham-controlled, parallel-group study. J Affect Disord 2020;274:93-102.

29. Begemann MJ, Brand BA, Curčić-Blake B, Aleman A, Sommer IE. Efficacy of non-invasive brain stimulation on cognitive functioning in brain disorders: a meta-analysis. Psychol Med 2020. doi: 10.1017/S0033291720003670. [Epub ahead of print]

30. Nejati V, Salehinejad MA, Nitsche MA, Najian A, Javadi AH. Transcranial direct current stimulation improves executive dysfunctions in ADHD: implications for inhibitory control, interference control, working memory, and cognitive flexibility. J Atten Disord 2020;24:1928-1943.

31. Salehinejad MA, Ghanavai E, Rostami R, Nejati V. Cognitive control dysfunction in emotion dysregulation and psychopathology of major depression (MD): Evidence from transcranial brain stimulation of the dorsolateral prefrontal cortex (DLPFC). J Affect Disord 2017;210:241-248.

32. Goldstein RZ, Volkow ND. Dysfunction of the prefrontal cortex in addiction: neuroimaging findings and clinical implications. Nat Rev Neurosci 2011;12:652-669.

33. Alizadehgoradel J, Nejati $V$, Sadeghi Movahed F, Imani S, Taherifard M, Mosayebi-Samani M, et al. Repeated stimulation of the dorsolateral-prefrontal cortex improves executive dysfunctions and craving in drug addiction: a randomized, double-blind, parallel-group study. Brain Stimul 2020; 13:582-593.

34. Schluter RS, Daams JG, van Holst RJ, Goudriaan AE. Effects of non-invasive neuromodulation on executive and other cognitive functions in addictive disorders: a systematic review. Front Neurosci 2018;12:642.

35. Luigjes J, Segrave R, de Joode N, Figee M, Denys D. Efficacy of invasive and non-invasive brain modulation interventions for addiction. Neuropsychol Rev 2019;29:116-138.

36. Shahbabaie A, Hatami J, Farhoudian A, Ekhtiari H, Khatibi A, Nitsche MA. Optimizing electrode montages of transcranial direct current stimulation for attentional bias modification in early abstinent methamphetamine users. Front Pharmacol 2018;9:907.

37. Bajbouj M, Aust S, Spies J, Herrera-Melendez AL, Mayer SV, Peters M, et al. PsychotherapyPlus: augmentation of cognitive behavioral therapy (CBT) with prefrontal transcranial direct current stimulation (tDCS) in major depressive disorder-study design and methodology of a multicenter double-blind randomized placebo-controlled trial. Eur Arch Psychiatry Clin Neurosci 2018;268:797-808.

38. Brunoni AR, Valiengo L, Baccaro A, Zanão TA, de Oliveira JF, Goulart A, et al. The sertraline vs. electrical current therapy for treating depression clinical study: results from a factorial, randomized, controlled trial. JAMA Psychiatry 2013;70:383-391.

39. Koganemaru S, Fukuyama $\mathrm{H}$, Mima T. Two is more than one: how to combine brain stimulation rehabilitative training for functional recovery? Front Syst Neurosci 2015;9:154.

40. Nejati V, Salehinejad MA, Shahidi N, Abedin A. Psychological intervention combined with direct electrical brain stimulation (PIN-CODES) for treating major depression: a pre-test, post-test, follow-up pilot study. Neurol Psychiatry Brain Res 2017;25: 15-23.

41. Li W, Howard MO, Garland EL, McGovern P, Lazar M. Mindfulness treatment for substance misuse: a systematic review and meta-analysis. J Subst Abuse Treat 2017;75:62-96.

42. Kabat-Zinn J, Hanh TN. Full catastrophe living: using the wisdom of your body and mind to face stress, pain, and illness. New York, NY:Delta Trade Paperbacks;2009. p.95-105.

43. Josipovic Z. Duality and nonduality in meditation research. Conscious Cogn 2010;19:1119-1121.

44. Garland EL, Froeliger B, Howard MO. Mindfulness training targets neurocognitive mechanisms of addiction at the attention-appraisal-emotion interface. Front Psychiatry 2014;4:173.

45. Himelstein S. Mindfulness-based substance abuse treatment for incarcerated youth: a mixed method pilot study. Int J Transpers Stud 2011;30:1-10.

46. Britton WB, Bootzin RR, Cousins JC, Hasler BP, Peck T, Shapiro SL. The contribution of mindfulness practice to a multicomponent behavioral sleep intervention following substance abuse treatment in adolescents: a treatment-development study. Subst Abus 2010;31:86-97.

47. Hunter MA, Lieberman G, Coffman BA, Trumbo MC, Armenta $\mathrm{ML}$, Robinson $\mathrm{CSH}$, et al. Mindfulness-based training with transcranial direct current stimulation modulates neuronal resource allocation in working memory: a randomized pilot study with a nonequivalent control group. Heliyon 2018;4: e00685.

48. Robinson CD, Waters AJ, Kang N, Sofuoglu M. Neurocognitive function as a treatment target for tobacco use disorder. Curr Behav Neurosci Rep 2017;4:10-20.

49. Badran BW, Austelle CW, Smith NR, Glusman CE, Froeliger B, Garland EL, et al. A double-blind study exploring the use of transcranial direct current stimulation (tDCS) to potentially 
enhance mindfulness meditation (E-Meditation). Brain Stimul 2017;10:152-154.

50. Monnart A, Vanderhasselt MA, Schroder E, Campanella S, Fontaine $\mathrm{P}$, Kornreich C. Treatment of resistant depression: a pilot study assessing the efficacy of a tDCS-mindfulness program compared with a tDCS-relaxation program. Front Psychiatry 2019;10:730.

51. Witkiewitz K, Stein ER, Votaw VR, Wilson AD, Roos CR, Gallegos SJ, et al. Mindfulness-based relapse prevention and transcranial direct current stimulation to reduce heavy drinking: a double-blind sham-controlled randomized trial. Alcohol Clin Exp Res 2019;43:1296-1307.

52. Himelstein S, Saul S. Mindfulness-based substance abuse treatment for adolescents: a 12-session curriculum. New York, NY:Routledge;2015. p.15.

53. Brewin CR, Smart L. Working memory capacity and suppression of intrusive thoughts. I Behav Ther Exp Psychiatry 2005;36:61-68.

54. Kane MJ, Conway ARA, Miura TK, Colflesh GJH. Working memory, attention control, and the N-back task: a question of construct validity. J Exp Psychol Learn Mem Cogn 2007;33: 615-622.

55. Demakis GJ. A meta-analytic review of the sensitivity of the Wisconsin Card Sorting Test to frontal and lateralized frontal brain damage. Neuropsychology 2003;17:255-264.

56. Lejuez CW, Read JP, Kahler CW, Richards JB, Ramsey SE, Stuart GL, et al. Evaluation of a behavioral measure of risk taking: the Balloon Analogue Risk Task (BART). J Exp Psychol Appl 2002;8:75-84.

57. Peterson E, Welsh MC. The development of hot and cool executive functions in childhood and adolescence: are we getting warmer? In: Goldstein S, Naglieri J, editors. Handbook of executive functioning. New York, NY:Springer;2014. p.45-65.

58. Franken IH, Hendriksa VM, van den Brink W. Initial validation of two opiate craving questionnaires the obsessive compulsive drug use scale and the desires for drug questionnaire. Addict Behav 2002;27:675-685.

59. Roche N, Geiger M, Bussel B. Mechanisms underlying transcranial direct current stimulation in rehabilitation. Ann Phys Rehabil Med 2015;58:214-219.

60. Unal G, Bikson M. Transcranial direct current stimulation (tDCS). In: Krames E, Hunter Peckham P, Rezai A, editors. Neuromodulation: comprehensive textbook of principles, technologies, and therapies. Cambridge:Academic Press;2018. p.1589-1610.

61. Thair H, Holloway AL, Newport R, Smith AD. Transcranial direct current stimulation (tDCS): a beginner's guide for design and implementation. Front Neurosci 2017;11:641.

62. Sreeraj VS, Shivakumar V, Bose A, Abhiram PN, Agarwal SM, Chhabra $\mathrm{H}$, et al. A functional domain based approach in neurocognitive rehabilitation with transcranial direct current stimulation: a case report. Clin Psychopharmacol Neurosci 2019;17:125-129.

63. Koob GF, Volkow ND. Neurobiology of addiction: a neurocircuitry analysis. Lancet Psychiatry 2016;3:760-773.

64. Garavan H. Insula and drug cravings. Brain Struct Funct 2010;214:593-601.

65. da Silva MC, Conti CL, Klauss J, Alves LG, do Nascimento Cavalcante HM, Fregni F, et al. Behavioral effects of transcranial direct current stimulation (tDCS) induced dorsolateral prefrontal cortex plasticity in alcohol dependence. J Physiol Paris 2013;107:493-502.

66. Nakamura-Palacios EM, de Almeida Benevides MC, da Penha Zago-Gomes M, de Oliveira RW, de Vasconcellos VF, de Castro LN, et al. Auditory event-related potentials (P3) and cognitive changes induced by frontal direct current stimulation in alcoholics according to Lesch alcoholism typology. Int J Neuropsychopharmacol 2012;15:601-616.

67. Fernández-Serrano MJ, Lozano O, Pérez-García M, VerdejoGarcía A. Impact of severity of drug use on discrete emotions recognition in polysubstance abusers. Drug A/cohol Depend 2010;109:57-64.

68. Spronk DB, van Wel JH, Ramaekers JG, Verkes RJ. Characterizing the cognitive effects of cocaine: a comprehensive review. Neurosci Biobehav Rev 2013;37:1838-1859.

69. Baldacchino A, Balfour DJ, Passetti F, Humphris G, Matthews K. Neuropsychological consequences of chronic opioid use: a quantitative review and meta-analysis. Neurosci Biobehav Rev 2012;36:2056-2068.

70. Garland EL. Restructuring reward processing with MindfulnessOriented Recovery Enhancement: novel therapeutic mechanisms to remediate hedonic dysregulation in addiction, stress, and pain. Ann N Y Acad Sci 2016;1373:25-37.

71. Priddy SE, Howard MO, Hanley AW, Riquino MR, FribergFelsted K, Garland EL. Mindfulness meditation in the treatment of substance use disorders and preventing future relapse. neurocognitive mechanisms and clinical implications. Subst Abuse Rehabil 2018;9:103-114. 